\title{
A 54-year-old Woman with Myelofibrosis and Massive Hemothorax Due to Primary Extramedullary Hematopoiesis of the Pleura
}

Michael Karass ${ }^{1}$, Katherine Linder ${ }^{1}$, Anup Agarwal ${ }^{1}$, Alexandra Budhai ${ }^{2}$, Yasmin Yusuf ${ }^{2}$, Oleg Epelbaum 1

1. Internal Medicine, Westchester Medical Center, Valhalla, USA 2. Pathology, Westchester Medical Center, Valhalla, USA

Corresponding author: Michael Karass, karass.michael@gmail.com

\begin{abstract}
Extramedullary hematopoiesis, which represents ectopic blood cell production, is usually an incidental finding accompanying hematologic pathology. The liver and spleen are the most common sites of extramedullary hematopoiesis, but thoracic involvement is likewise observed. Pleural effusions in the setting of intrathoracic extramedullary hematopoiesis have been attributed to mechanical interactions between the pleural surface and neighboring paravertebral masses consisting of hematopoietic tissue. Rupture of these highly vascularized lesions into the adjacent pleural space has been the putative mechanism in cases complicated by hemothorax. Histologically proven instances of islets of extramedullary hematopoiesis occurring on the pleural surface itself are exceedingly rare. Our case of a patient with myelofibrosis and massive pleural effusion is only the third such example described in the literature and the second to result in a confirmed hemothorax requiring surgery. As expected, technetium-99m sulfur (Tc-99m sulfur) colloid scanning accurately localized sites of extramedullary hematopoiesis in our patient, and there was a salutary response to radiation therapy.
\end{abstract}

Received 09/17/2018

Review began 09/24/2018 Review ended 11/30/2018 Published 12/03/2018

๑) Copyright 2018

Karass et al. This is an open access article distributed under the terms of the Creative Commons Attribution License CC-BY 3.0., which permits unrestricted use, distribution, and reproduction in any medium, provided the original author and source are credited.
Categories: Cardiac/Thoracic/Vascular Surgery, Oncology, Pulmonology

Keywords: pleural, myelofibrosis, hematopoiesis, hemothorax, extramedullary, radiation

\section{Introduction}

Extramedullary hematopoiesis (EMH) is a well-recognized feature of hematopoietic disorders such as myelofibrosis and thalassemia. Reported thoracic manifestations of EMH have included paravertebral mass lesions [1], diffuse alveolar hemorrhage [2], and pleural effusion [3]. There are numerous descriptions of hemothorax resulting from EMH [4-12], but in these cases, the bleeding was attributable to the rupture of the paravertebral foci of EMH into the adjacent pleural space. In contrast, the direct involvement of pleural tissue with EMH implants is an extremely rare finding confirmed histologically only thrice previously to our knowledge [13-15]. Only one of these three cases [14] was complicated by massive hemothorax. We report the second such occurrence.

\section{Case Presentation}

A 54-year-old woman with a 20-year history of myelofibrosis (MF) being treated with ruxolitinib and complicated by pancytopenia and massive splenomegaly initially presented to an outside hospital with progressive dyspnea and generalized weakness. Laboratory evaluation at that hospital revealed worsened anemia compared to the patient's baseline. Chest imaging demonstrated a large left pleural effusion for which she underwent thoracentesis. She was eventually transferred to our institution for further management.

On presentation to our institution, the patient's blood pressure was $131 / 85 \mathrm{mmHg}$, pulse 90 beats/min, and respiratory rate 18 breaths $/ \mathrm{min}$. She had a temperature of $98.1 \mathrm{~F}$ and an oxygen saturation of $98 \%$ while breathing supplemental oxygen via a nasal cannula at $2 \mathrm{~L} / \mathrm{min}$. Mild respiratory distress was apparent. On cardiac examination, she had a grade $2 / 6$ systolic ejection murmur. Lung examination was notable for decreased breath sounds at the left base with dullness to percussion. Other pertinent findings were pallor, palpable splenomegaly, and lower extremity edema.

Abnormal laboratory values included a leukocytosis of $18.4 \mathrm{k} / \mathrm{mm} 3$ (normal range $4.8-10.8 \mathrm{k} / \mathrm{mm} 3$ ) with an increase in myeloid precursors, hemoglobin of $5.6 \mathrm{~g} / \mathrm{dl}$ (normal range $12-16 \mathrm{~g} / \mathrm{dL}$ ), thrombocytopenia of $77 \mathrm{k} / \mathrm{mm} 3$ (normal range 160-410 k/mm3), and lactate dehydrogenase (LDH) of $882 \mathrm{U} / \mathrm{L}$ (normal range 125220 U/L). Her coagulation parameters were unremarkable. Portable chest X-ray showed complete opacification of the left hemithorax with a rightward displacement of the trachea (Figure 1). Urgent placement of a left chest tube yielded grossly bloody pleural fluid consisting of a red blood cell (RBC) count of $1,415,000 / \mathrm{mm} 3$ and an $\mathrm{LDH}$ of $1555 \mathrm{U} / \mathrm{L}$. The approximate pleural fluid hematocrit of $14 \%$ was $>50 \%$ of serum hematocrit consistent with hemothorax. She received three units of packed RBCs over the ensuing 


\section{Cureus}

two days but surgical intervention was not pursued. There was no evidence of malignancy by cytology or flow cytometry. The chest tube was removed on hospital Day 9, as there was no active drainage with an interval decrease of pleural effusion and stable serum hemoglobin. Over the subsequent two days, however, her serum hemoglobin again dropped from 8.0g/dL to 6.5g/dL. On hospital Day 11, computed tomography (CT) angiogram of the chest, abdomen, and pelvis was performed to localize a source of bleeding. The CT angiogram showed a large, complex fluid collection in the left pleural space measuring 11.6 x $9.6 \mathrm{~cm}$ in cross-section with a hyperdense focus compatible with active extravasation (Figure 2). The patient was taken to the operating room for urgent exploration. Approximately 2.5 liters of blood and blood clots were surgically evacuated. There was no evidence of a discrete bleeding source, but there was diffuse pleural oozing. Decortication of a thin fibrous peel was performed. She was extubated 24 hours

postoperatively and transferred to the general medical floor after discontinuation of all chest tubes. A hematoxylin \& eosin stained section of the patient's pleura biopsied intraoperatively revealed maturing erythroid and myeloid cells with small clusters of erythroid and myeloid precursors (Figure $3 A$ ). Also present were atypical and dysplastic megakaryocytes that were highlighted by CD61 immunostaining (Figure 3B). The finding of trilineage hematopoiesis in a pleural sample is diagnostic of EMH at that location. Nuclear imaging with Technetium-99m sulfur (Tc-99m sulfur) colloid demonstrated diffusely increased uptake in the chest bilaterally, right greater than left, consistent with intrathoracic EMH (Figure 4). As expected, there was decreased activity within the bone marrow compatible with the patient's history of myelofibrosis.

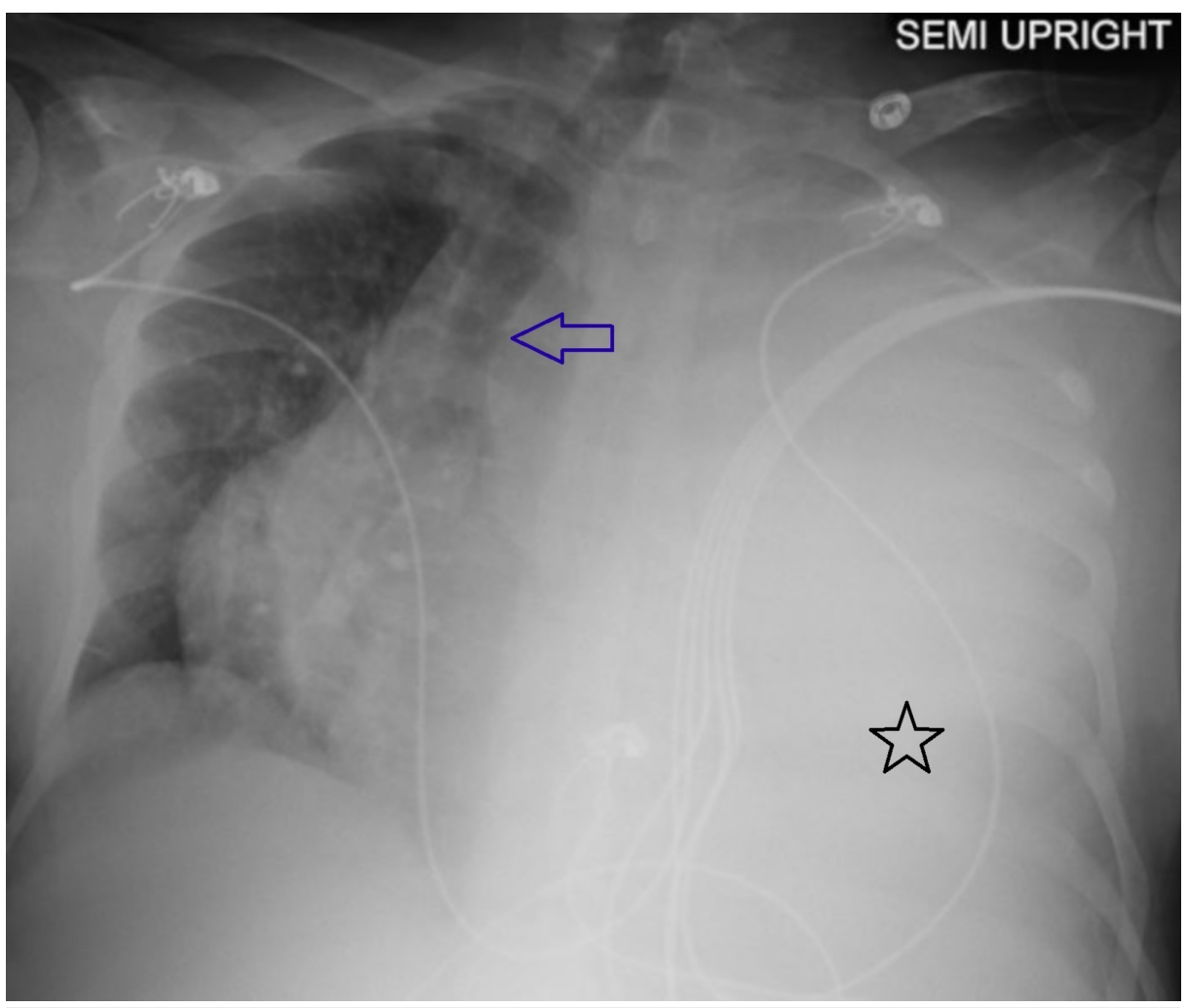

\section{FIGURE 1: Left pleural effusion on chest X-ray}

Portable plain radiograph of the chest showing a massive left pleural effusion (black star) with contralateral displacement of trachea (blue arrow) 


\section{Cureus}
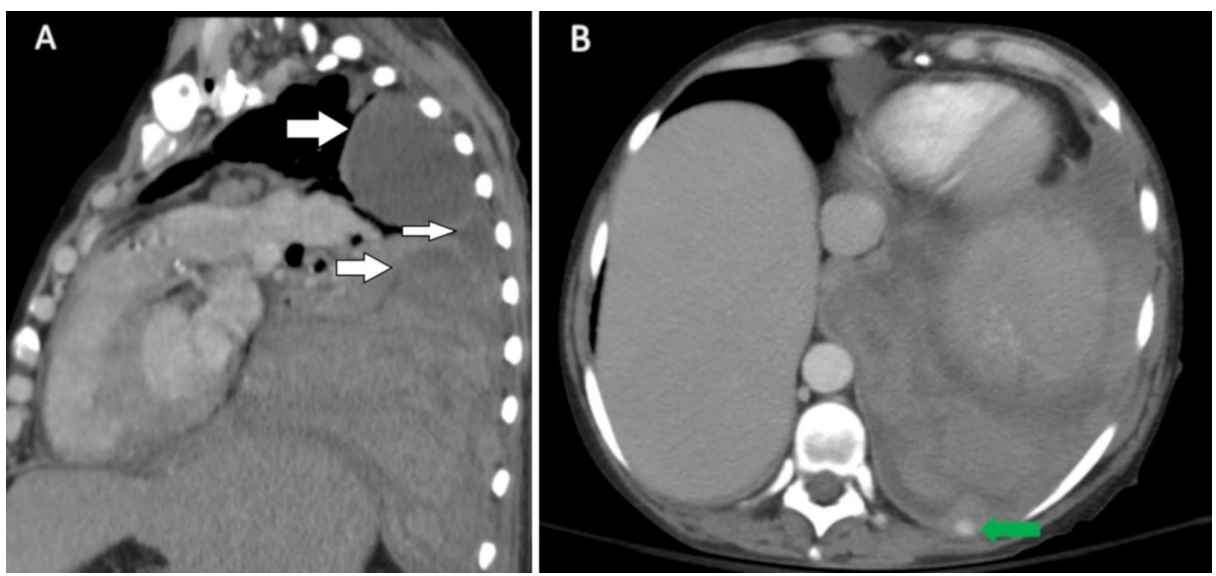

FIGURE 2: Multi-loculated pleural effusion and active bleeding on CT angiogram

A) Coronal view from the computed tomography (CT) angiogram of the chest showing a multi-loculated pleural fluid collection in the left hemithorax (white arrows). B) Axial image from the CT angiogram showing extravasation of contrast (green arrow) compatible with active bleeding

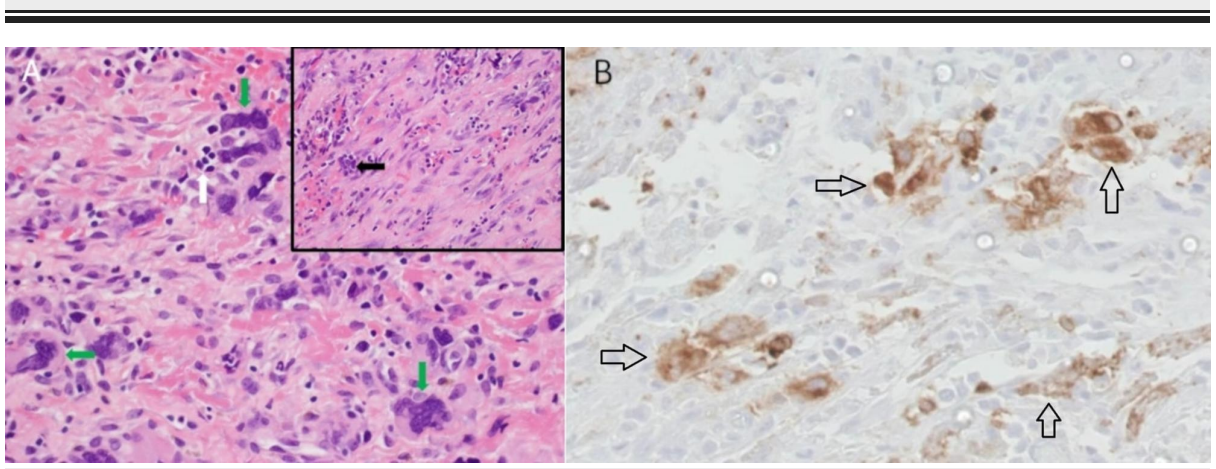

FIGURE 3: Pleural biopsy with megakaryocytes seen with hematoxylin \& eosin and immunostaining

A) Pleural biopsy section showing multiple megakaryocytes (green arrows) as well as an erythroid precursor (white arrow). Inset shows a different section containing a myeloid precursor (black arrow) (hematoxylin \& eosin, original magnification $x 400$, inset $x$ 100). B) Pleural tissue immuno-staining for CD61 (black arrows), which highlights megakaryocytes (original magnification $x 400$ ). 


\section{Cureus}

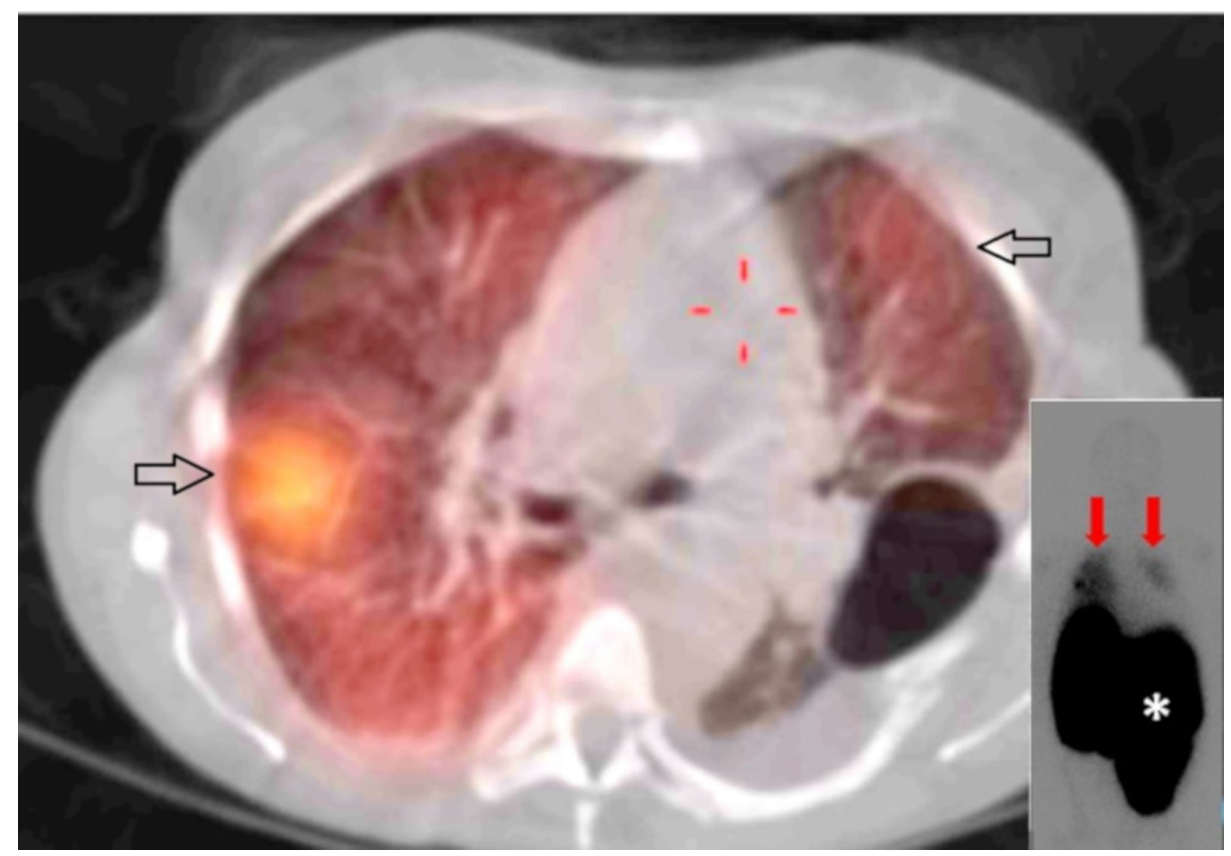

\section{FIGURE 4: Increased uptake on SPECT/CT}

Technetium-99m sulfur (Tc-99m sulfur) colloid single photon emission computed tomography/CT (SPECT/CT) axial fusion image showing increased uptake in the thorax (black arrows). Planar scintigraphy images (inset lower right) likewise demonstrate increased uptake in both the right and left hemithorax (red arrows) while highlighting the massive splenomegaly characteristic of myelofibrosis (asterisk)

\section{Discussion}

EMH is the production of blood cell elements outside of the bone marrow. It can be a compensatory mechanism in bone marrow replacement pathology, such as MF, in hemoglobinopathies such as thalassemia and sickle cell disease, in red cell dyscrasias such as hereditary spherocytosis, and in many other hematologic conditions. The usual sites of EMH are the liver and spleen, but it has been encountered in virtually all organs [15]. Intrathoracic EMH is a rare but well-described finding; the most common locations are the paravertebral space within the posterior mediastinum, the ribs, and the lung parenchyma [16]. It is typically an incidental discovery in a patient with known hematopoietic pathology. Clinical and radiographic suspicion for this diagnosis can be supported by the increased uptake of technetium-99m sulfur colloid on nuclear scanning, which is a substance absorbed selectively by reticuloendothelial cells [17]. Tissue sampling of EMH sites is considered inadvisable due to the propensity of these densely vascularized structures for bleeding. Foci of EMH are exquisitely sensitive to radiation therapy (RT), which is the typical management strategy [18].

Within the already rare category of intrathoracic EMH, pleural involvement is exceedingly uncommon. The majority of existing reports describe patients with thalassemia who developed hemothorax [4-5,7-12] and, therefore, presented with dyspnea in contrast to uncomplicated cases of intrathoracic EMH, which tend to be asymptomatic. Based on information from reports with surgical or autopsy findings [4-5,8-12], pleural hemorrhage is most often the result of rupture of paravertebral, juxtapleural foci of EMH into the pleural space with resultant bleeding. This pathogenetic mechanism could be considered a secondary form of pleural EMH as opposed to the current case, which could be thought of as primary pleural EMH because lesions were actually detected in the pleural surface itself and were the likely source of bleeding. If one considered cases with identifiable pleural implants to be a separate entity, then this patient belongs to a unique category described in detail only twice previously [13-14]. The first such description [13] dates back to 1967 and reports a very similar patient: a 65-year-old woman with constitutional symptoms and a left pleural effusion. Thoracentesis was unsuccessful, so a pleural biopsy was performed, which revealed hematopoietic elements. Further evaluation led to the diagnosis of myelofibrosis, and the patient expired 18 months following her original presentation. At autopsy, the visceral pleural surface was found to be covered by nodules and plaques histologically composed of hematopoietic cells. The other case report [14] was published in 1993 and described a 73-year-old woman with known myelofibrosis who presented with dyspnea and a right pleural effusion. Initial thoracentesis yielded a serosanguinous exudative fluid not consistent with hemothorax. The pleural biopsy was non-diagnostic at that time. When the effusion recurred two weeks later, doxycycline pleurodesis was performed via tube thoracostomy, following which her symptoms and chest X-ray worsened. Emergent exploratory surgery resulted in the evacuation of approximately 2 liters of clotted blood. Decortication and pleural biopsy were performed, and the histological findings were consistent with EMH. She required radiation therapy and additional operative 
explorations to control the bleeding but subsequently succumbed to superimposed sepsis. Autopsy findings revealed multi-organ EMH.

The current patient is the third detailed description of primary EMH of the pleura, with the publication frequency of these three cases being approximately once every 25 years. Like the two before her, she is of female gender and has underlying myelofibrosis. Particularly instructive is the comparison with the more recent case from 1993, which also resulted in pleural hemorrhage. That patient initially presented with a pleural effusion that was not a hemothorax, and frank blood filled the pleural space only after subsequent chest tube placement and chemical pleurodesis. Although the results of the initial thoracentesis performed at the referring hospital in our patient are not available, it is reasonable to surmise based on the lack of urgent pleural intervention at that facility that the initial pleural fluid sample was not consistent with hemothorax. It is likely that the first discovery of hemothorax in our case occurred following pleural drain placement at our institution. This pattern suggests that pleural manipulation may be required to convert non-bleeding pleural EMH to a source of active hemorrhage. It appears that a subset of patients with pleural EMH carries asymptomatic implants, some present with pleural effusion that is not hemothorax, and yet others develop hemorrhage triggered by intervention. The possibility that many patients with hematopoietic disorders may harbor unrecognized foci of pleural EMH is illustrated by the 1967 case mentioned above as well as a patient included in a 2003 series from the Mayo Clinic [15] in which pleural EMH was incidentally discovered in the setting of chronic lymphocytic leukemia. Table 1 summarizes the three primary pleural EMH cases, including the current one, which has been described in detail.

\begin{tabular}{|c|c|c|c|c|c|}
\hline Citation/Year & Age/Gender & Underlying Disease & Hemothorax & Prior Pleural Intervention & Outcome \\
\hline Anton et al (1967) [13] & $65 / F$ & MF & $\mathrm{N}$ & N/A & Death $^{*}$ \\
\hline Kuperschmid et al (1993) [14] & 73/F & MF & $\mathrm{Y}$ & Pleurodesis & Death ${ }^{\#}$ \\
\hline Present Case (2018) & $54 / F$ & MF & $\mathrm{Y}$ & Drainage & Alive $^{\$}$ \\
\hline \multicolumn{6}{|c|}{$\begin{array}{l}\text { TABLE 1: Summary of the three primary pleural extramedullary hematopoiesis cases described ir } \\
\text { detail in the literature }\end{array}$} \\
\hline \multicolumn{6}{|c|}{$\mathrm{MF}=$ myelofibrosis, $\mathrm{N} / \mathrm{A}=$ not applicable } \\
\hline \multicolumn{6}{|c|}{ "Patient died within a year of presentation } \\
\hline \multicolumn{6}{|c|}{ "Patient died during index hospitalization } \\
\hline SPatient alive three years after inde & admission & & & & \\
\hline
\end{tabular}

The patient underwent $200 \mathrm{cGy}$ of RT over four days to the left pleura for pleural EMH based on findings on the nuclear scan, with a resolution of hemothorax, and was discharged to a rehabilitation facility. She is currently alive about three years after the event, making her the first survivor of massive hemothorax due to primary pleural EMH since the patient described in 1993 expired during the index hospitalization.

\section{Conclusions}

EMH of the thorax is a well-described entity, but clinically overt involvement of the pleural space is rare. Hemothorax in the setting of EMH has been primarily reported in the setting of rupture of juxtapleural paravertebral EMH into the pleural space. Instances of hemothorax caused by primary pleural EMH are exceedingly uncommon, and bleeding in such cases may be precipitated by pleural intervention. Tc-99m sulfur colloid nuclear scanning can be used to confirm the suspicion of intrathoracic EMH, thus obviating the need to sample these richly vascularized lesions. EMH tissue is highly radiosensitive, so RT is the treatment of choice in symptomatic cases.

\section{Additional Information \\ Disclosures}

Human subjects: Consent was obtained by all participants in this study. Conflicts of interest: In compliance with the ICMJE uniform disclosure form, all authors declare the following: Payment/services info: All authors have declared that no financial support was received from any organization for the submitted work. Financial relationships: All authors have declared that they have no financial relationships at present or within the previous three years with any organizations that might have an interest in the submitted work. Other relationships: All authors have declared that there are no other 


\section{References}

1. Georgiades CS, Neyman EG, Francis IR, Sneider MB, Fishman EK: Typical and atypical presentations of extramedullary hematopoiesis. AJR Am J Roentgenol. 2002, 179:1239-1243. 10.2214/ajr.179.5.1791239

2. Ozbudak IH, Shilo K, Hale S, Aguilera NS, Galvin JR, Franks TJ: Alveolar airspace and pulmonary artery involvement by extramedullary hematopoiesis: a unique manifestation of myelofibrosis. Arch Pathol Lab Med. 2008, 142:99-103.

3. Bartlett RP, Greipp PR, Tefferi A, Cupps RE, Mullan BP, Trastek VF: Extramedullary hematopoiesis manifesting as a symptomatic pleural effusion. Mayo Clin Proc. 1995, 70:1161-1164. 10.4065/70.12.1161

4. Smith PR, Manjoney DL, Teitcher JB, Choi KN, Braverman AS: Massive hemothorax due to intrathoracic extramedullary hematopoiesis in a patient with thalassemia intermedia. Chest. 1988, 94:658-660. 10.1378/chest.94.3.658

5. Chu KA, Lai RS, Lee CH, Lu JY, Chang HC, Chiang HT: Intrathoracic extramedullary hematopoiesis complicated by massive haemothorax in alpha-thalassemia. Thorax. 1999, 54:466-468. 10.1136/thx.54.5.466

6. Xiros N, Economopoulos T, Papageorgiou E, Mantzios G, Raptis S: Massive hemothorax due to intrathoracic extramedullary hematopoiesis in a patient with hereditary spherocytosis. Ann Hematol. 2001, 80:38-40.

7. Tassiopoulos S, Konstantopoulos K, Rombos Y, Aessopos A: Hemothorax due to extramedullary erythropoietic masses. Ann Thorac Surg. 2004, 77:323-324. 10.1016/S0003-4975(03)00754-9

8. Chute DJ, Fowler DR: Fatal hemothorax due to rupture of an intrathoracic extramedullary hematopoietic nodule. Am J Forensic Med Pathol. 2004, 25:74-77. 10.1097/01.paf.0000113859.48471.49

9. Pornsuriyasak P, Suwatanapongched T, Wangsuppasawad N, Ngodngamthaweesuk M, Angchaisuksiri P: Massive hemothorax in a beta-thalassemic patient due to spontaneous rupture of extramedullary hematopoietic masses: diagnosis and successful treatment. Respir Care. 2006, 51:272-276.

10. Tantraworasin A, Saeteng S: Massive hemothorax due to intrathoracic extramedullary hematopoiesis in a patient with beta thalassemia hemoglobin E disease. J Med Assoc Thai. 2013, 96:866-869.

11. Chu KA, Hsu CW, Lin MH, Lin SJ, Huang YL: Recurrent spontaneous massive hemothorax from intrathoracic extramedullary hematopoiesis resulting in respiratory failure. J Formos Med Assoc. 2015, 114:282-284. 10.1016/j.jfma.2012.02.006

12. Ma SH, Hu SY, Hsieh MS: Intrathoracic extramedullary hematopoiesis related spontaneous hemothorax. QJM. 2015, 108:989. 10.1093/qjmed/hcv143

13. Anton HC, Ferguson JB, Lewis GP: Pleural extramedullary haematopoiesis in myelosclerosis . Postgrad Med J. 1967, 43:428-432. 10.1136/pgmj.43.500.428

14. Kupferschmid JP, Shahian DM, Villanueva AG: Massive hemothorax associated with intrathoracic extramedullary hematopoiesis involving the pleura. Chest. 1993, 103:974-975. 10.1378/chest.103.3.974

15. Koch CA, Li CY, Mesa RZ, Tefferi A: Nonhepatosplenic extramedullary hematopoiesis: associated diseases, pathology, clinical course, and treatment. Mayo Clin Proc. 2003, 78:1223-1233. 10.4065/78.10.1223

16. Roberts AS, Shetty AS, Mellnick VM, Pickhardt PJ, Bhalla S, Menias CO: Extramedullary hematopoiesis: radiological imaging features. Clin Radiol. 2016, 71:807-814. 10.1016/j.crad.2016.05.014

17. Bronn LJ, Paquelet JR, Tetalman MR: Intrathoracic extramedullary hematopoiesis: appearance on 99mTc sulfur colloid marrow scan. AJR Am J Roentgenol. 1980, 134:1254-1255. 10.2214/ajr.134.6.1254

18. Weinschenker P, Kutner JM, Salvajoli JV, Hanriot RM, Ribeiro AF, Capelozzi VL, Giglio AD: Wholepulmonary low-dose radiation therapy in agnogenic myeloid metaplasia with diffuse lung involvement. Am J Hematol. 2002, 69:277-280. 10.1002/ajh.10075 